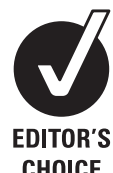

CHOICE

Pennine Acute Hospitals NHS Trust, Greater Manchester, UK

\section{Correspondence to}

Dr Jimmy K Limdi, Consultant Gastroenterologist, Pennine Acute Hospitals NHS Trust, Manchester BL9 7TD, UK; jimmy.limdi@pat.nhs.uk

Received 15 July 2010 Accepted 21 November 2010 Published Online First 17 January 2011

\title{
Acute severe ulcerative colitis: timing is everything
}

\author{
Stephen R Gulliford, Jimmy K Limdi
}

\section{ABSTRACT}

The idiopathic inflammatory bowel diseases comprise mainly two types of intestinal disorder, Crohn disease and ulcerative colitis. The clinical course is marked by exacerbations and remissions that occur spontaneously in response to treatment or intercurrent illness. The disease affects approximately 240000 patients in the UK. Acute severe ulcerative colitis is a medical emergency; prompt effective treatment at the point of admission can avoid significant morbidity and be potentially life-saving. Although such patients need specialist management, it is imperative that emergency care physicians are aware of the important principles of management of this condition to achieve successful outcomes. Corticosteroids remain the cornerstone of initial therapy, but a third of patients will fail to respond, and further management involves critical and timely decisions on whether to use rescue therapy in the form of ciclosporin or infliximab without compromising the health or safety of the patient or to offer timely surgery. The evidence base for the choices for optimal management of this condition is presented.

\section{INTRODUCTION}

The idiopathic inflammatory bowel diseases (IBDs) comprise mainly two types of intestinal disorderCrohn disease (CD) and ulcerative colitis (UC)characterised by gastrointestinal inflammation. The clinical course is marked by exacerbations and remissions that occur spontaneously or in response to treatment or intercurrent illnesses. The exact aetiology remains unknown, but accumulating evidence suggests that it is the result of complex interactions between the genetic make up of the individual with environmental and mucosal immunity. $^{1}$

The disease affects approximately 240000 people in the UK, with an estimated prevalence of 400 per 100000 populations. The condition is most prevalent in developed nations, but with rising incidence in developing countries. The two conditions are remarkably similar but with important differences. ${ }^{2}$

CD can affect any part of the gastrointestinal tract, although it most commonly affects the ileum and colon, often discontinuously. Inflammation is often transmural and may be associated with granulomas, strictures or fistulae. UC, on the other hand, is typically a mucosal inflammatory disease involving the rectum and affecting part of or the entire colon (pancolitis) in an uninterrupted fashion. The peak onset is between 15 and 30 years of age. Patients with IBD are at risk of primary sclerosing cholangitis, ankylosing spondylitis and psoriasis. ${ }^{1}$

The majority of patients with IBD are managed in the outpatient setting, but a small though significant number of patients will have acute severe and sometimes life-threatening complications requiring urgent and immediate medical intervention. The second UK National IBD audit conducted in 2008 revealed that management of patients with acute severe UC is still suboptimal. Although it is imperative that a gastroenterologist with expertise in IBD manages these patients, emergency care physicians should also be aware of the important principles of management of this condition to achieve successful outcomes.

\section{BACKGROUND}

Although most patients will present with mild to moderate UC, $10 \%$ of patients present with severe disease. Approximately $15 \%$ of patients will develop a severe flare during the course of their lifetime. Natural history studies suggest that $10-40 \%$ of patients will undergo a colectomy during the course of their disease. ${ }^{3} 4$

Acute severe UC may be defined by the Truelove and Witts criteria: a stool frequency of six or more per day, with a temperature of $>37.8^{\circ} \mathrm{C}$, pulse rate $>90$ beats $/ \mathrm{min}$, large amount of blood in stools, haemoglobin concentration $<10.5 \mathrm{~g} / \mathrm{dl}$ or erythrocyte sedimentation rate $>30 \mathrm{~mm} / \mathrm{h}^{5}$ (table 1 ). More recent practice position statements of the European Crohn's and Colitis Organisation and American College of Gastroenterology define severe colitis similarly as passage of six or more stools with evidence of systemic toxicity (tachycardia, fever, anaemia or elevated erythrocyte sedimentation rate). ${ }^{67}$ Acute fulminant UC implies progression of inflammation beyond the mucosa and is associated with systemic symptoms. These patients require immediate hospitalisation for intensive management because of the seriousness of their illness. It is also advisable to admit less-sick patients who have failed to respond to 2 weeks of oral corticosteroid therapy. As an initial attack of severe UC may be more dangerous than subsequent flares, there should be a lower threshold to admit patients presenting with bloody diarrhoea. Although there has been a reduction in the mortality from acute severe UC with the adoption of more aggressive monitoring, ${ }^{5}$ such as the use of intravenous steroid and improved and timely surgery, this condition is regrettably still associated with measurable mortality. $^{8} 9$

To optimise the management of acute severe colitis, meticulous attention should be given to confirming the diagnosis, establishing the disease activity, site and extent, and selecting and monitoring the appropriate treatment. A multidisciplinary team led by a gastroenterologist experienced in the management of IBD is best suited to the management of such patients. Throughout the disease course, the patient must be kept fully 
Table 1 Truelove and Witts criteria for severe ulcerative colitis

\begin{tabular}{llll}
\hline Criterion & Mild & Severe & Fulminant \\
\hline Stools per day & $<4$ & $>6$ & $>10$ \\
Blood in stool & Small/intermittent & Frequent & Continuous \\
Temperature & Normal & $>37.5^{\circ} \mathrm{C}$ & $>37.5^{\circ} \mathrm{C}$ \\
Pulse & Normal & $>90$ & $>90$ \\
Haemoglobin & $>75 \%$ of & $<75 \%$ of & Transfusion \\
Erythrocyte sedimentation rate & $<30$ & normal & required \\
& & $>30$ & $>30$ \\
\hline
\end{tabular}

informed about the disease and be involved in clinical decision making.

\section{Initial management}

Patients with acute severe UC should be admitted to a specialist gastroenterology ward for joint medical, surgical and nursing care. Criteria for admission are defined in table 1.

Early nutritional assessment, an IBD specialist nurse if available, and a counsellor may prove particularly helpful, and also a stoma therapist if a patient is deemed likely to need surgery. A detailed daily clinical assessment should be performed, and an accurate stool chart maintained. Electrolytes and C-reactive protein (CRP) should be checked daily. An abdominal plain radiograph should be obtained to exclude signs of toxic dilatation, as discussed below. This should be done daily for as long as the patient fulfils the criteria for acute severe colitis or in the event of acute deterioration.

It is important to exclude other causes of diarrhoea and bleeding, summarised in box 1 . Therefore a stool examination for microscopy, culture and Clostridium difficile toxin analysis is imperative. Infective colitis should be excluded in all patients, but especially those with abrupt onset of diarrhoea, vomiting or fever, those with an epidemic or contact history, and those with a recent history of foreign travel. Non-steroidal anti-inflammatory drugs may trigger a relapse (flare) of UC, but equally may also be responsible for de novo colitis, which typically remits upon withdrawal of the drugs. Radiation colitis can occur in patients with previous abdominal or pelvic radiation, and ischaemic colitis should be considered in patients with established (or risk factors for) vascular disease and is usually marked by abrupt onset of abdominal pain with bloody diarrhoea.

Endoscopic assessment with a limited, unprepared sigmoidoscopy and biopsies with minimal air insufflation should be performed by an experienced operator to exclude $C$ difficile and cytomegalovirus. ${ }^{710}$ A full colonoscopy is unnecessary and may be associated with an increased risk of complications. Infective colitis is characterised by crypt architectural preservation at histopathology, whereas crypt distortion is an important differentiating feature of UC.

Serial abdominal radiographs provide vital information in the management of acute UC. The presence of mucosal islands, referred to as 'thumb printing' when seen, is a predictor of failure of medical treatment. Severe active disease is characterised by the presence of deep mucosal ulceration or coarse mucosal nodularity. The presence of faecal residue indicates uninflamed colonic mucosa. Proximal constipation is often seen in left-sided or distal colitis, which often exacerbates distal disease and paradoxically requires laxatives in addition to standard treatment. Measurement of the transverse colonic and caecal diameter gives important clues to the presence of colonic dilatation (diameter $>5.5 \mathrm{~cm}$ ) and impending toxic dilatation. An erect chest radiograph or a lateral decubitus abdominal film may confirm colonic perforation.
Box 1 Differential diagnoses of diarrhoea with rectal bleeding

- Idiopathic Inflammatory bowel disease

- Crohn disease

- Ulcerative colitis

- Indeterminate colitis

- Infection

- Clostridium difficile

- Campylobacter

- Salmonella

- Shigella

- Escherichia coli (0157:H7NTEC)

- Yersinia

- Aeromonas

- Listeria

- Amoebiasis

- Tuberculosis

- Schistosomiasis

- Herpes and cytomegalovirus infection in immunocompromised

- Drug induced

- Non-steroidal anti-inflammatory medication

- Antibiotics

- Gold

- Miscellaneous

- Ischaemic colitis

- Radiation colitis

- Vasculitis (polyarteritis nodosa, Behcet's disease)

- Diverticulitis

- Colorectal cancer

\section{Supportive therapy}

Although the cornerstone of management of acute severe UC remains the use of corticosteroids, meticulous attention to supportive management is vital for success of any pharmacological intervention.

Most patients will need intravenous fluids with correction of electrolyte imbalance; in particular, serum potassium should be maintained above $4 \mathrm{mmol} / \mathrm{l}$ as hypokalaemia may predispose to colonic dilatation, with most patients requiring routine replacement of $60 \mathrm{mmol} / \mathrm{l}$ potassium in $24 \mathrm{~h}$. A blood transfusion should be considered for patients with haemoglobin concentration $<10 \mathrm{~g} / \mathrm{dl}$. Oral iron therapy is probably best avoided, with anecdotal reports suggesting that it can aggravate mucosal inflammation through oxidative stress from oxygen free radicals, the 'Fenton' reaction. ${ }^{11}$

There is no proven advantage to the routine use of bowel rest or parenteral nutrition, although nutritional assessment and optimisation of nutritional status is important for successful outcome. $^{1213}$

Patients with acute severe UC are at high risk of venous thromboembolism, and efforts to minimise this with the use of compression stockings and low molecular weight heparin are appropriate. $^{7} 14$

The routine use of intravenous antibiotics offers no therapeutic advantage in uncomplicated colitis, although they may be appropriate in patients with incipient perforation or toxic megacolon or in those in whom infection is suspected. ${ }^{15} 16$ There is no evidence that 5-aminosalicylates have any effect on disease activity in the setting of acute severe UC and may in fact be 
associated with a paradoxical worsening of disease, therefore treatment with these agents should be withheld until the acute episode has resolved. In addition, antidiarrhoeal and anticholinergic agents and opiates should be avoided, as they may prove harmful by decreasing colonic motility and increasing the risk of colonic dilatation and toxic megacolon. ${ }^{6} 7$

The principles of supportive therapy in acute severe UC are summarised in box 2 .

\section{Corticosteroid therapy}

Intravenous corticosteroid therapy in the form of hydrocortisone (300-400 mg daily in divided doses) or methylprednisolone (40-60 mg/day) should be administered. No real advantage can be gained from giving higher doses. Some clinicians may also use prednisolone $20 \mathrm{mg}$ or hydrocortisone $100 \mathrm{mg}$ in $200 \mathrm{ml}$ water given rectally via a soft catheter and intravenous set twice daily with the patient in the left lateral position, but the value of this regimen is unproven.

Most patients will respond to intravenous steroid therapy and may be switched to oral prednisolone $(40-60 \mathrm{mg}$ daily with taper over the next 2-3 months). Approximately $30 \%$ of patients will fail to respond to this strategy and are termed 'steroid refractory'. ${ }^{17}$ Patients who do not respond to steroid therapy by day $3-5$ pose a significant therapeutic challenge, emphasising the importance of contingency planning and the collaborative efforts of a multidisciplinary approach involving the gastroenterologist, an experienced surgeon and the patient. Therapeutic options are generally limited to continued rescue medical therapy with ciclosporin or infliximab or colectomy. Clinical and laboratory variables are often used to make decisions about early failure of corticosteroid therapy. ${ }^{17}{ }^{18}$ One of the most useful algorithms is the Travis criterion (box 3); at day 3 of corticosteroid therapy, patients who have a stool frequency of more than eight per day or a stool frequency of three per day plus CRP $>45 \mathrm{mg} / \mathrm{dl}$ have an $85 \%$ likelihood of requiring colectomy during the admission. ${ }^{19}$

Other studies have also demonstrated the importance of a time-bound approach in determining steroid unresponsiveness.

Box 2 Principles of management (supportive therapy) in acute severe ulcerative colitis

- Admit to a specialist gastroenterological ward for multidisciplinary care (joint medical and surgical)

- Daily clinical assessment and examination

- Daily electrolytes and C-reactive protein

- Nutritional assessment and optimisation of nutritional status

- Bowel rest is of no proven benefit

- Flexible sigmoidoscopy (preferably unprepared) at the earliest opportunity

- Fluid resuscitation as required

- Correction of electrolyte abnormalities

- Blood transfusion if haemoglobin concentration drops below $10.5 \mathrm{~g} / \mathrm{dl}$

- Prophylaxis against venous thromboembolism with low molecular weight heparin

- Empirical antibiotics are of no benefit, except where infection is suspected, or there is incipient perforation or toxic megacolon.

- Avoid anticholinergics, opiates and antidiarrhoeal agents, as they increase the risk of colonic dilatation and perforation
Box 3 Travis criterion-prediction of colectomy after

failure of intravenous corticosteroid therapy by day 3

( $85 \%$ chance of colectomy if criteria fulfilled. $)^{19}$

- Stool frequency $>8$ times per day

Or

- Stool frequency of $>3$ per day

Plus

- $\mathrm{CRP}>45 \mathrm{mg} / \mathrm{dl}$

Lindgren and colleagues prospectively studied 97 episodes of admissions with acute severe UC and suggested a mathematical model to predict colectomy: (Number of stools in $24 \mathrm{~h}$ $+0.14 \times \operatorname{CRP}(\mathrm{mg} / \mathrm{l}))>8$ predicted a $72 \%$ colectomy rate. ${ }^{20}$

Ho and colleagues retrospectively studied 167 episodes of acute severe $U^{21}$ and identified a number of criteria with a score predicting colectomy or need for rescue therapy in steroid-unresponsive patients. Number of stools (score 1-4), hypoalbuminaemia $<30 \mathrm{~g} / \mathrm{l}$ (score 1) and colonic dilatation $>5.5 \mathrm{~cm}$ (score 4) were noted as predictors, with $85 \%$ of patients with a score of 4 or more needing colectomy or second-line therapy. ${ }^{21}$

The decision regarding colectomy or continued salvage medical therapy and choice of such treatment (infliximab or ciclosporin) should be made within the first 3-5 days to ensure favourable outcome. Although medical therapy for steroidrefractory UC has expanded in recent years with the availability of ciclosporin and infliximab, surgery remains the only curative option. We discuss below the data supporting the use of both drugs and comparison with long-term outcomes with restorative proctocolectomy and ileal pouch-anal anastomosis (IPAA).

\section{Ciclosporin in acute severe colitis}

Ciclosporin A (CyA) is an inhibitor of the transcription factor, nuclear factor kappa B of activated $T$ cells and acts mainly by attenuation of production of interleukin-2 by activated $T$ cells. It can be an effective rescue therapy in acute severe UC when used judiciously in experienced hands.

The efficacy of CyA in acute steroid-refractory UC was demonstrated definitively in the landmark study by Lichtiger et al in 1994 when nine of 11 patients with steroid-refractory UC who received CyA $(4 \mathrm{mg} / \mathrm{kg})$ as a continuous intravenous infusion improved, whereas all nine patients who received placebo showed no improvement. ${ }^{22}$ Three of 11 and four of nine patients underwent colectomy in the CyA and placebo groups, respectively. This trial is often criticised for small patient numbers, but it is important to point out that the study was stopped early by the safety committee because the investigators had demonstrated a clear difference in efficacy in the CyA group.

There have been at least 30 open label trials since then demonstrating clinical response in at least two-thirds of patients, with the use of CyA and systematic reviews supporting its efficacy as rescue therapy in acute steroid-refractory UC. ${ }^{23-25}$

CyA has also shown similar efficacy to intravenous methylprednisolone for the induction of remission in acute $U C$ in a randomised trial, ${ }^{26}$ suggesting that CyA monotherapy may be an option for patients with acute severe UC who have contraindications to steroid therapy, such as steroid psychosis, avascular necrosis and severe osteoporosis. It is also worth mentioning here that a number of patients for whom 
monotherapy with corticosteroid or CyA failed responded to combination therapy, suggesting that combination therapy may be more effective.

The $4 \mathrm{mg} / \mathrm{kg}$ dose used by the initial and subsequent trials was associated with a number of side effects and toxicity. Van Assche and colleagues in a landmark randomised controlled trial compared $4 \mathrm{mg} / \mathrm{kg}$ with $2 \mathrm{mg} / \mathrm{kg}$ intravenous CyA and showed equal efficacy for the two treatment groups in treating severe steroid-refractory UC. ${ }^{27}$ Response rates at day 8 were similar in the two groups at $82 \%$ and $83 \%$, respectively. Other studies have substantiated this claim using low-dose CyA as monotherapy without initial or concurrent steroids followed by early introduction of azathioprine (AZA), demonstrating that low-dose CyA is associated with minimal morbidity and no mortality. ${ }^{28} 29$

Although the data presented above would suggest that CyA is an attractive therapeutic option in experienced hands, an important measure of successful therapy is the avoidance of, or time to, colectomy. Several studies have attempted to describe the long-term efficacy in patients treated with CyA for steroidrefractory UC. Campbell et al conducted a retrospective review of 76 patients treated over 7 years and found that 56 (74\%) achieved initial remission, while 20 (26\%) underwent surgery during the same admission. ${ }^{29}$ Of the 56 patients in remission, $65 \%$ relapsed in 1 year and $58 \%$ underwent a colectomy at 7 years. In a study by Actis et al, $60 \%$ of patients with steroidrefractory UC responded to CyA, and, of these initial responders, $60 \%$ were colectomy-free at 1 year and $35 \%$ were colectomy-free at 7 years. $^{30}$

Long-term data on colectomy-free survival suggest a high failure rate after CyA salvage therapy. ${ }^{31}{ }^{32}$ Shah et al showed an $86 \%$ response rate with intravenous CyA (36 of 42 patients), with 25 of these 42 patients avoiding surgery at 23 months follow-up. Nineteen of these 25 initial responders were noted to be colectomy-free at a median of 81 months, and an overall $45 \%$ of initial patients did not require surgery after 75 months. ${ }^{31}$

In one study, concurrent immunosuppression with thiopurines (AZA/mercaptopurine) appears to have an important effect on long-term efficacy of CyA. Patients receiving CyA and AZA had a $66 \%$ probability of avoiding colectomy at follow-up (5.5 years) as opposed to $40 \%$ in the group that received CyA alone. Among initial responders to CyA, a $71 \%$ response was noted in the combination group as opposed to $55 \%$ in those who received CyA alone. In this study $32 \%$ started AZA with CyA therapy, 28\% received AZA a mean of 15 months before CyA treatment, $8 \%$ had AZA upon stopping ciclosporin, and the rest started AZA 8 months after stopping CyA. ${ }^{33}$

Data on patients already taking immunomodulators at the time of CyA therapy for acute severe UC have shown a high likelihood of requiring colectomy. ${ }^{32}$ In the study by Moskovitz et al, $77 \%$ of patients taking AZA on admission with acute severe UC required colectomy; $88 \%$ needed colectomy within 12 months of receiving CyA in this group. This was in contrast with $35 \%$ of patients who were given AZA during their admission when administered CyA therapy and needed colectomy. $^{32}$

It seems fairly clear from the available data that the long-term outcome for fulminant UC is improved by the introduction of thiopurine immunomodulatory therapy and several months of oral ciclosporin as bridging therapy when using CyA as rescue treatment.

Is it possible to identify predictive factors for response to ciclosporin? Cacheux and colleagues ${ }^{34}$ identified that a body temperature $>37.5^{\circ} \mathrm{C}$ and pulse $>90$ beats/minute with CRP $>45 \mathrm{mg} / \mathrm{dl}$ predicted colectomy in CyA-treated patients.
Colectomy rates were noted to be $0.22,0.47,0.55$ and 0.90 when 0, 1, 2 and 3 of these factors were present. Endoscopic lesions in the form of deep ulceration or mucosal abrasion were independent risk factors for colectomy, with colectomy rates of $72 \%$ at 6 months in patients who had severe endoscopic lesions as against $42 \%$ in those who did not have them.

One of the most important considerations with regard to CyA therapy in acute severe UC is its potential for toxicity. Large case series have reported a significant toxicity profile, with serious infections in $5 \%$ of patients and mortality in $1-3 \% .^{29} 30 \quad 35-37$ Major adverse events related to CyA therapy include nephrotoxicity $(6.3 \%)$, seizures $(3.6 \%)$, anaphylaxis $(0.9 \%)$ and death $(1.8 \%){ }^{37}$ Minor but important adverse events include parasthaesia, hypertension, hypertrichosis, headache, minor infections, hyperkalaemia, hypomagnesaemia and gingival swelling.

Many of the reported toxicities can be avoided. The use of prophylactic sulfamethoxazole and trimethoprim may prevent Pneumocystis carinii infection. ${ }^{36} 37$ The risk of seizures can be mitigated by adequate control of blood pressure, correction of hypomagnesaemia, adequate monitoring of CyA concentrations, and using the drug with caution in patients with low serum cholesterol. ${ }^{38}$ The risk of renal toxicity may be reduced by using a lower dose, although cases of irreversible renal impairment have been reported. ${ }^{39}$ Seizure risk in patients with hypomagnesaemia or hypocholesterolaemia may be reduced if the oral micro emulsion form of CyA is used at a dose of $5 \mathrm{mg} / \mathrm{kg} /$ day, which appears to be equivalent to the intravenous dose, providing a possibly safer alternative in such patients. ${ }^{40-42}$ Treatment with CyA does not appear to increase operative mortality in patients subsequently requiring restorative proctocolectomy. ${ }^{43} \mathrm{~A}$ $36 \%$ postoperative complication rate has been reported with CyA, which is similar to the complication rates for patients undergoing colectomy and not treated previously with CyA. ${ }^{44}$

Practical considerations when using CyA deserve mention here. The initial dose is $2 \mathrm{mg} / \mathrm{kg} /$ day intravenously with a target CyA concentration of between 150 and $250 \mathrm{ng} / \mathrm{ml}$ by monoclonal assay. Patients who respond will be converted to an oral dose twice the intravenous dose and administered orally in divided doses twice daily with a trough concentration of $100-200 \mathrm{ng} / \mathrm{ml}$. An exit strategy in the form of AZA or 6-mercaptopurine should be initiated during hospitalisation and continued in the outpatient setting. Prophylaxis for $P$ carinii should be initiated for the duration of dual therapy for 3-6 months, after which patients may be able to stop CyA therapy and continue AZA.

The well-timed use of CyA in the management of acute severe UC is safe and effective with impressive outcomes in experienced hands.

\section{Tacrolimus}

Tacrolimus (FK506) is an alternative calcineurin inhibitor with a similar side effect profile to CyA. It can be administered by the oral route and has a reliable bioavailability. Data on tacrolimus in steroid-refractory UC are limited, with a single randomised controlled trial of 63 patients showing an improvement in $68 \%$ of patients randomised to receive tacrolimus as opposed to $10 \%$ response in the placebo group. ${ }^{45}$ Tacrolimus rescue therapy is recognised by European Crohn's and Colitis Organisation guidelines. ${ }^{7}$

\section{Infliximab in acute severe UC}

Infliximab is a chimeric IgG1 monoclonal antibody that specifically targets free and membrane-bound tumour necrosis factor- $\alpha$. It was initially licensed as treatment for moderate to 
severely active CD and approved subsequently for use in moderate to severely active UC after two large placebocontrolled trials, the Active UC trial (Act I \& II), demonstrated efficacy. ${ }^{46}$ Several other studies have examined infliximab as an effective rescue therapy in acute severe UC. Järnero et al randomised 45 patients with acute severe steroid-refractory UC to a single infusion of infliximab $(5 \mathrm{mg} / \mathrm{kg}$ ) or placebo 4 days after initiating steroids. Seven of 24 patients in the infliximab group $(29 \%)$ and 14 of 21 patients in the placebo group had a colectomy within 3 months of randomisation. ${ }^{47}$ No long-term follow-up data were provided.

Lees et al reported that approximately a third of patients treated with infliximab went on to have a colectomy during the acute admission. ${ }^{48}$ In a study by Kohn et al of 83 patients with steroid-refractory UC treated with infliximab, 15\% underwent colectomy in 2 months and 58 patients avoided colectomy at a median 23 months follow-up. The investigators noted that two infusions appeared more effective than a single dose. ${ }^{49}$ Jakobovits et al from Oxford reported that $47 \%$ of patients had avoided colectomy after their first infusion at a median of 140 days, and $17 \%$ of these sustained a steroid-free remission. ${ }^{50}$ A Belgian study reported their experience with acute severe UC treated with infliximab. Single-dose infliximab was used in 84 patients, and 37 patients received a three-dose induction at 0.2 and 6 weeks. Two-thirds of these patients were receiving immunomodulators. An early response was seen in $65 \%$ of patients, the majority of whom were younger than nonresponders. A p-ANCA (protoplasmic-staining antineutrophil cytoplasmic antibodies) positivity and/or ASCA (anti-Saccharomyces cerevisiae mannan antibodies)-negative serotype was associated with a suboptimal response. ${ }^{51} \mathrm{~A}$ meta-analysis by Gisbert and colleagues concluded that infliximab may improve short- and long-term remission rates in patients with moderate to severe UC. ${ }^{52}$ It would appear from the evidence available that infliximab may benefit up to two-thirds of patients with acute severe steroid-refractory UC in the short term; it probably works less well in the sickest of patients. ${ }^{48}$

Toxicity is a concern with infliximab as it is with CyA. Areas of concern are infections, lymphoma, drug-induced liver injury and demyelinating disease. It is also to be avoided in patients with severe congestive cardiac failure.

Does infliximab therapy impact on surgical outcomes and perioperative complications? The evidence is conflicting, with some earlier studies in patients with CD reporting no increase in early postoperative complications, ${ }^{53} 54$ and other investigators reporting a risk of early readmission, intra-abdominal abscess and postoperative complications. ${ }^{55}$ Studies in patients with UC have reported an increase in postoperative complications in patients treated preoperatively with infliximab and CyA but not in those treated with infliximab. ${ }^{56-58}$

It seems clear that there is no role for combination therapy with infliximab and CyA in acute steroid-refractory UC. The combination is associated with higher morbidity and an up to $80 \%$ rate of infectious and surgical complications. ${ }^{57}$ Further, CyA and infliximab as rescue therapy for each other cannot be recommended, with a recent study demonstrating an increased risk of serious side effects ( $16 \%$ patients) including one death. ${ }^{59}$

The decision on whether to use CyA or infliximab is largely based on physician experience, and, although most gastroenterologists are comfortable with infliximab because of its widespread use in $\mathrm{CD}$, the available data for sicker patients are more robust for CyA, especially for patients with severe steroidrefractory UC. For patients with their first episode of acute severe UC who are naïve to thiopurine therapy, there are sufficient data suggesting that the use of CyA followed by the introduction of AZA while the patient is still in hospital with 3 months of oral bridging therapy with oral CyA is effective as discussed above. In patients already receiving AZA, infliximab is more successful. $^{30} \mathrm{~A}$ head-to-head trial comparing infliximab with CyA would provide much needed data and clarity to help resolve this important debate. The results of the GETAID and CONSTRUCT trials investigating this issue are eagerly awaited.

\section{Colectomy for acute severe UC}

That UC is a surgically curable disease is an irrefutable fact. Surgical removal of the colon, proximal rectum and distal rectal mucosa provides a definitive means of treating acute colitis, thereby eliminating diseased tissue and any future risk of recurrence and malignancy. Surgery decreases mortality and may improve quality of life, but carries a significant risk of perioperative complications and functional outcomes that may not always be acceptable to all patients. Emergency indications for surgery include toxic dilatation, haemorrhage, intestinal perforation or systemic instability, whereas a severe attack of UC 'unresponsive to medical therapy' represents an urgent indication, a term itself that needs more precise definition. ${ }^{60}$ Delaying surgery in patients with worsening symptoms or development of clinical instability can lead to dire consequences, with the risk of adverse outcomes including death. ${ }^{8}$ This underpins the importance of contingency planning with an 'exit strategy' in steroid-refractory patients by day 5 of admission.

Restorative proctocolectomy has evolved as a procedure of choice for patients with acute severe UC. Subtotal colectomy and ileostomy is the procedure of choice in this situation, with the surgeon's objective being to perform a low-risk operation without pelvic dissection, and a more definitive procedure, namely a staged reconstructive procedure with IPAA or definitive total proctocolectomy, is performed when the patient's nutritional status has improved and immunosuppressive drugs are no longer being taken. Typically this is a three-stage procedure taking $\sim 3$ months to complete.

Colectomy does carry some risk of complications and morbidity, albeit less than the futile continuation of medical therapy in unstable patients. Reported complications include abscess (11.6\%), fistulas (4.2\%) and bacteraemia/sepsis (7.9\%). ${ }^{61}$ Pouchitis is the most important complication of pouch reconstruction, which may occur in up to $50 \%$ of patients within 10 years, with other pouch-related disorders, such as pouch dysfunction and cuffitis, also being described. ${ }^{62}$ Functional outcomes after an IPAA are generally good, with a median bowel frequency of six motions per day. Intermittent urgency (23\%), evacuation difficulties (12\%) and intermittent soiling at night (52\% male, $32 \%$ female patients) have been reported. ${ }^{63}$ Decreased fertility after pouch surgery is also increasingly being recognised, with the risk of female infertility being reported as high as threefold. ${ }^{64}$ It may be appropriate therefore for women to delay restorative IPAA until after childbearing years.

Patients should be carefully counselled, with equal weight being given to the positive aspects of surgery, such as cure of UC and elimination of the risk of colorectal cancer, and potential complications and functional outcomes when the data on surgical outcomes are presented. The collaborative efforts of a multidisciplinary team approach (gastroenterologist, surgeon, psychologist, stoma therapist) cannot be overemphasised.

\section{Novel therapies}

The treatment of severe steroid-refractory UC remains suboptimal, and more effective treatment options are needed. Novel 
approaches to treatment have been tested in a number of uncontrolled trials. A single infusion of basilixumab (antibody to proinflammatory cytokine IL-2), for example, showed promise, with a $90 \%$ remission at 8 weeks, but the effect was not sustained. ${ }^{65} 66$

In an open label study of visilixumab (IgG2 humanised antibody to CD3 expressed on human $\mathrm{T}$ lymphocytes) in 32 patients, a 30 -day remission rate of $40 \%$ was reported. ${ }^{67}$ Phase III trials have been limited owing to concerns regarding safety and lack of efficacy. Several other agents such as cyclophosphamide, thalidomide, interferon $\beta$, phosphatidylcholine and leucocyte apheresis are being studied and may present attractive treatment options in the future. ${ }^{68-72}$

\section{CONCLUSION}

Acute severe UC is a medical emergency requiring hospitalisation for intensive monitoring and therapy under a gastroenterologist with experience in managing such patients. The cornerstone of treatment is the use of intravenous corticosteroids, but $\sim 30 \%$ will prove refractory to therapy and require the expertise of a multidisciplinary approach with the patient, gastroenterologist, colorectal surgeon, stoma therapist, nutritionist and, if available, a psychologist. The critical decision for patients who have not responded to corticosteroids by day 5 is whether it is appropriate to push the limits of medical 'rescue therapy' without compromising the health or safety of the patient or offer timely surgery. Both ciclosporin and infliximab appear safe and effective when used appropriately and in experienced hands. Ciclosporin is an attractive option in AZA-naïve patients who can be effectively bridged to purine immunomodulator maintenance therapy. In patients already receiving thiopurine therapy, CyA is less effective, and it may be more appropriate to use infliximab. Infliximab does appear less effective in very sick patients. Colectomy is a definitive means of treating acute colitis, thereby eliminating diseased tissue and any future risk of recurrence and malignancy, but carries important functional and psychological implications, which deserve due consideration. The improved understanding of the immunopathogenesis of gut inflammation and repair has translated into bolder attempts at the manipulation of the cytokine profile, which now enter medical territory. A key and unanswered question is whether this will translate into timely and meaningful control of disease. The jury is still out. Meanwhile, the clock starts ticking the moment a patient with acute severe colitis is hospitalised, and, with respect to investigation and treatment, timing is everything.

\section{Current research questions}

- Infliximab or ciclosporin for acute severe ulcerative colitis? Results from two head-to-head trials comparing efficacy are awaited.

- Controlled data from trials evaluating novel and experimental therapies are needed.

- Will genotype predict disease course, response to treatment, or the timing and need for colectomy?

-What is the optimal timing for colectomy?

- Additional data on efficacy and safety of current therapies in the short and long term are needed.

\section{Main messages}

- Intravenous corticosteroid therapy forms the cornerstone of treatment for patients admitted with acute severe ulcerative colitis.

- Early liaison with colorectal surgeons and for steroid nonresponders by day 3-also a stoma therapist and psychologist-is appropriate in anticipation of the need for colectomy if required.

- A time-bound approach for steroid non-responders between days 3 and 5 assessing predictors of response is critical to favourable outcomes. Infliximab, ciclosporin or surgery?

- Delaying or denying colectomy to the patient 'failing' medical treatment may lead to adverse outcomes.

\section{Key references}

Roberts SE, Williams JG, Yeates D, et al. Mortality in patients with and without colectomy admitted to hospital for ulcerative colitis and Crohn's disease: record linkage studies. BMJ 2007;335:1033. An important study emphasising the importance of timely surgery and adverse outcomes with inappropriate delay in considering colectomy.

- Grainge MJ, West J, Card TR. Venous thromboembolism during active disease and remission in inflammatory bowel disease: a cohort study. Lancet 2010;375:657-63. A thoughtprovoking paper demonstrating high-risk venous thromboembolism in patients with inflammatory bowel disease.

- Travis SP, Farrant JM, Ricketts C, et al. Predicting outcome in severe ulcerative colitis. Gut 1996;38:905-10. Landmark study that prognosticates 'high-risk' patients and led to a paradigm shift in clinical decision making in acute severe ulcerative colitis.

- Lichtiger S, Present DH, Kornbluth A, et al. Cyclosporine in severe ulcerative colitis refractory to steroid therapy. N Engl J Med 1994;330:1841-5. Sentinel study demonstrating successful outcomes with cyclosporine $A$ in acute severe ulcerative colitis.

- Rutgeerts P, Sandborn WJ, Feagan BG, et al. Infliximab for induction and maintenance therapy for ulcerative colitis. N Engl J Med 2005;353:2462-76. Landmark randomised controlled trial demonstrating efficacy of infliximab in moderate to severe ulcerative colitis.

\section{SELF-ASSESSMENT OUESTIONS (TRUE (T)/FALSE (F): ANSWERS AFTER THE REFERENCES)}

1. In the assessment of acute severe UC a colonoscopy is mandatory.

2. 5-Aminosalicylate therapy should be prescribed or continued in all patients with acute severe UC admitted to hospital.

3. Ciclosporin therapy is not the best option for steroidrefractory patients with acute severe UC already taking azathioprine.

4. Combination therapy with infliximab and ciclosporin A in acute severe UC is more effective and associated with lower morbidity. 
5. Despite the availability of modern and effective therapy for steroid-refractory acute severe UC, timely consideration of colectomy is appropriate.

Competing interests None.

Provenance and peer review Not commissioned; externally peer reviewed.

\section{REFERENCES}

1. Abraham C, Cho JH. Inflammatory bowel disease. N Engl J Med 2009;361:2066-78.

2. Rubin GP, Hungin AP, Kelly PJ, et al. Inflammatory bowel disease: epidemiology and management in an English general practice population. Aliment Pharmacol Ther 2000;14:1553-9.

3. Solberg IC, Lygren I, Jahnsen J, et al; IBSEN Study Group. Clinical course during the first 10 years of ulcerative colitis: results from a population-based inception cohort (IBSEN Study). Scand J Gastroenterol 2009;44:431-40.

4. Farmer RG, Easley KA, Rankin GB. Clinical patterns, natural history, and progression of ulcerative colitis. A long-term follow-up of 1116 patients. Dig Dis Sci 1993;38:1137-46.

5. Truelove SC, Witts LJ. Cortisone in ulcerative colitis; final report on a therapeutic trial. Br Med J 1955;2:1041-8.

6. Kornbluth A, Sachar DB; Practice Parameters Committee of the American College of Gastroenterology. Ulcerative colitis practice guidelines in adults: American College of Gastroenterology, Practice Parameters Committee. Am J Gastroenterol 2010:105:501-23.

7. Travis SPL, Stange EF, Lemman M, et al. European evidence based consensus on the management of ulcerative colitis: current management. J Crohn's Colitis 2008:2:24-62

8. Roberts SE, Williams JG, Yeates D, et al. Mortality in patients with and without colectomy admitted to hospital for ulcerative colitis and Crohn's disease: record linkage studies. BMJ 2007;335:1033.

9. Sonnenberg A. Time trends of mortality from Crohn's disease and ulcerative colitis. Int J Epidemiol 2007;36:890-9.

10. Maher MM, Nassar Ml. Acute cytomegalovirus infection is a risk factor in refractory and complicated inflammatory bowel disease. Dig Dis Sci 2009;54:2456-62.

11. Gasche C, Berstad A, Befrits R, et al. Guidelines on the diagnosis and management of iron deficiency and anemia in inflammatory bowel diseases. Inflamm Bowel Dis 2007:13:1545-53.

12. Dickinson RJ, Ashton MG, Axon AT, et al. Controlled trial of intravenous hyper alimentation and total bowel rest as an adjunct to the routine therapy of acute colitis. Gastroenterology 1980:79:1199-204

13. McIntyre PB, Powell-Tuck J, Wood SR, et al. Controlled trial of bowel rest in the treatment of severe acute colitis. Gut 1986;27:481-5.

14. Grainge MJ, West J, Card TR. Venous thromboembolism during active disease and remission in inflammatory bowel disease: a cohort study. Lancet 2010;375:657-63.

15. Chapman RW, Selby WS, Jewell DP. Controlled trial of intravenous metronidazole as an adjunct to corticosteroids in severe ulcerative colitis. Gut 1986:27:1210-12.

16. Mantzaris GJ, Petraki K, Archavlis E, et al. A prospective randomized controlled trial of intravenous ciprofloxacin as an adjunct to corticosteroids in acute, severe ulcerative colitis. Scand J Gastroenterol 2001;36:971-4.

17. Turner D, Walsh CM, Steinhart AH, et al. Response to corticosteroids in severe ulcerative colitis: a systematic review of the literature and a meta-regression. Clin Gastroenterol Hepatol 2007:5:103-10.

18. Lennard-Jones JE, Ritchie JK, Hilder W, et al. Assessment of severity in colitis: a preliminary study. Gut 1975:16:579-84.

19. Travis SP, Farrant JM, Ricketts $C$, et al. Predicting outcome in severe ulcerative colitis. Gut 1996;38:905-10.

20. Lindgren SC, Flood LM, Kilander AF, et al. Early predictors of glucocorticosteroid treatment failure in severe and moderately severe attacks of ulcerative colitis. Eur $\mathrm{J}$ Gastroenterol Hepatol 1998;10:831-5.

21. Ho GT, Mowat C, Goddard CJ, et al. Predicting the outcome of severe ulcerative colitis: development of a novel risk score to aid early selection of patients for secondline medical therapy or surgery. Aliment Pharmacol Ther 2004;19:1079-87.

22. Lichtiger S, Present $\mathrm{DH}$, Kornbluth $\mathrm{A}$, et al. Cyclosporine in severe ulcerative colitis refractory to steroid therapy. N Engl J Med 1994;330:1841-5.

23. García-López S, Gomollón-García F, Pérez-Gisbert J. Cyclosporine in the treatment of severe attack of ulcerative colitis: a systematic review. Gastroenterol Hepatol 2005; 28:607-14.

24. Durai D, Hawthorne AB. Review article: how and when to use ciclosporin in ulcerative colitis. Aliment Pharmacol Ther 2005;22:907-16.

25. Shibolet O, Regushevskaya E, Brezis M, et al. Cyclosporine A for induction of remission in severe ulcerative colitis. Cochrane Database Syst Rev 2005;(1): CD004277.

26. D'Haens G, Lemmens L, Geboes K, et al. Intravenous cyclosporine versus intravenous corticosteroids as single therapy for severe attacks of ulcerative colitis. Gastroenterology 2001;120:1323-9.

27. Van Assche G, D'Haens G, Noman M, et al. Randomized, double-blind comparison of $4 \mathrm{mg} / \mathrm{kg}$ versus $2 \mathrm{mg} / \mathrm{kg}$ intravenous cyclosporine in severe ulcerative colitis. Gastroenterology 2003;125:1025-31.
28. Rayner CK, McCormack G, Emmanuel AV, et al. Long-term results of low-dose intravenous ciclosporin for acute severe ulcerative colitis. Aliment Pharmacol Ther 2003;18:303-8.

29. Campbell S, Travis S, Jewell D. Ciclosporin use in acute ulcerative colitis: a long-term experience. Eur J Gastroenterol Hepatol 2005;17:79-84.

30. Actis GC, Fadda M, David E, et al. Colectomy rate in steroid-refractory colitis initially responsive to cyclosporin: a long-term retrospective cohort study. BMC Gastroenterol 2007;7:13

31. Shah SB, Parekh NK, Hanauer SB, et al. Intravenous cyclosporine in severe steroid refractory ulcerative colitis: long term follow up. Gastroenterology 2008;134(Suppl 1):133 [1041].

32. Moskovitz DN, Van Assche G, Maenhout B, et al. Incidence of colectomy during long-term follow-up after cyclosporine-induced remission of severe ulcerative colitis. Clin Gastroenterol Hepatol 2006;4:760-5.

33. Cohen RD, Stein R, Hanauer SB. Intravenous cyclosporin in ulcerative colitis: a five-year experience. Am J Gastroenterol 1999;94:1587-92.

34. Cacheux W, Seksik P, Lemann M, et al. Predictive factors of response to cyclosporine in steroid-refractory ulcerative colitis. Am J Gastroenterol 2008;103:637-42.

35. Cullen G, Keegan D, O'Donoghue D. Safety and efficacy of cyclosporine therapy in inflammatory bowel disease. Am J Gastroenterol 2008;103:2654-5.

36. Arts $\mathbf{J}, D^{\prime}$ Haens $\mathrm{G}$, Zeegers $\mathrm{M}$, et al. Long-term outcome of treatment with intravenous cyclosporin in patients with severe ulcerative colitis. Inflamm Bowel Dis 2004; 10:73-8.

37. Sternthal MB, Murphy SJ, George J, et al. Adverse events associated with the use of cyclosporine in patients with inflammatory bowel disease. Am J Gastroenterol 2008:103:937-43.

38. Kornbluth A, Present DH, Lichtiger $\mathrm{S}$, et al. Cyclosporin for severe ulcerative colitis: a user's guide. Am J Gastroenterol 1997;92:1424-8.

39. Haslam N, Hearing SD, Probert CS. Audit of cyclosporin use in inflammatory bowe disease: limited benefits, numerous side-effects. Eur J Gastroenterol Hepatol 2000;12:657-60

40. Actis GC, Aimo G, Priolo G, et al. Efficacy and efficiency of oral microemulsion cyclosporin versus intravenous and soft gelatin capsule cyclosporin in the treatment of severe steroid-refractory ulcerative colitis: an open-label retrospective trial. Inflamm Bowel Dis 1998:4:276-9.

41. Navazo L, Salata H, Morales S, et al. Oral microemulsion cyclosporine in the treatment of steroid-refractory attacks of ulcerative and indeterminate colitis. Scand J Gastroenterol 2001:36:610-14.

42. Weber A, Fein F, Koch S, et al. Treatment of ulcerative colitis refractory to steroid therapy by oral microemulsion cyclosporine (Neoral). Inflamm Bowel Dis 2006; 12:1131-5.

43. Hyde GM, Jewell DP, Kettlewell MG, et al. Cyclosporin for severe ulcerative colitis does not increase the rate of perioperative complications. Dis Colon Rectum 2001:44:1436-40.

44. Pinna-Pintor M, Arese P, Bona R, et al. Severe steroid-unresponsive ulcerative colitis: outcomes of restorative proctocolectomy in patients undergoing cyclosporin treatment. Dis Colon Rectum 2000;43:609-13.

45. Ogata H, Matsui T, Nakamura M, et al. A randomised dose finding study of oral tacrolimus (FK506) therapy in refractory ulcerative colitis. Gut 2006;55:1255-62.

46. Rutgeerts $\mathbf{P}$, Sandborn WJ, Feagan BG, et al. Infliximab for induction and maintenance therapy for ulcerative colitis. N Engl J Med 2005;353:2462-76.

47. Järnerot G, Hertervig E, Friis-Liby I, et al. Infliximab as rescue therapy in severe to moderately severe ulcerative colitis: a randomized, placebo-controlled study. Gastroenterology 2005;128:1805-11.

48. Lees CW, Heys D, Ho GT, et al. Scottish Society of Gastroenterology Infliximab Group. A retrospective analysis of the efficacy and safety of infliximab as rescue therapy in acute severe ulcerative colitis. Aliment Pharmacol Ther 2007; $26: 411-19$

49. Kohn A, Daperno M, Armuzzi A et al. Infliximab in severe ulcerative colitis: short-term results of different infusion regimens and long-term follow-up. Aliment Pharmacol Ther 2007;26:747-56.

50. Jakobovits SL, Jewell DP, Travis SP. Infliximab for the treatment of ulcerative colitis: outcomes in Oxford from 2000 to 2006. Aliment Pharmacol Ther 2007:25:1055-60.

51. Ferrante $\mathbf{M}$, Vermeire $\mathrm{S}$, Katsanos $\mathrm{KH}$, et al. Predictors of early response to infliximab in patients with ulcerative colitis. Inflamm Bowel Dis 2007;13:123-8.

52. Gisbert JP, González-Lama Y, Maté J. Systematic review: Infliximab therapy in ulcerative colitis. Aliment Pharmacol Ther 2007;25:19-37.

53. Colombel JF, Loftus EV Jr, Tremaine WJ, et al. Early postoperative complications are not increased in patients with Crohn's disease treated perioperatively with infliximab or immunosuppressive therapy. Am J Gastroenterol 2004;99:878-83.

54. Marchal L, D'Haens G, Van Assche G, et al. The risk of post-operative complications associated with infliximab therapy for Crohn's disease: a controlled cohort study. Aliment Pharmacol Ther 2004;19:749-54.

55. Appau KA, Fazio WW, Shen B, et al. Use of infliximab within 3 months of ileocolonic resection is associated with adverse postoperative outcomes in Crohn's patients. $J$ Gastrointest Surg. 2008;12:1738-44.

56. Kunitake H, Hodin R, Shellito PC, et al. Perioperative treatment with infliximab in patients with Crohn's disease and ulcerative colitis is not associated with an increased rate of postoperative complications. J Gastrointest Surg 2008;12:1730-6. 
57. Schluender SJ, Ippoliti A, Dubinsky M, et al. Does infliximab influence surgical morbidity of ileal pouch-anal anastomosis in patients with ulcerative colitis? Dis Colon Rectum 2007:50:1747-53.

58. Selvasekar CR, Cima RR, Larson DW, et al. Effect of infliximab on short-term complications in patients undergoing operation for chronic ulcerative colitis. J Am Coll Surg 2007;204:956-62.

59. Maser EA, Deconda D, Lichtiger S, et al. Cyclosporine and infliximab as rescue therapy for each other in patients with steroid-refractory ulcerative colitis. Clin Gastroenterol Hepatol 2008:6:1112-16.

60. Teeuwen PH, Stommel MW, Bremers AJ, et al. Colectomy in patients with acute colitis: a systematic review. J Gastrointest Surg 2009;13:676-86.

61. Loftus EV Jr, Delgado DJ, Friedman HS, et al. Colectomy and the incidence of postsurgical complications among ulcerative colitis patients with private health insurance in the United States. Am J Gastroenterol 2008;103:1737-45.

62. Meagher AP, Farouk R, Dozois RR, et al. J ileal pouch-anal anastomosis for chronic ulcerative colitis: complications and long-term outcome in 1310 patients. Br J Surg 1998;85:800-3.

63. Berndtsson I, Lindholm E, Oresland T, et al. Long-term outcome after ilea pouch-anal anastomosis: function and health-related quality of life. Dis Colon Rectum 2007:50:1545-52

64. Waljee A, Waljee J, Morris AM, et al. Threefold increased risk of infertility: a meta-analysis of infertility after ileal pouch anal anastomosis in ulcerative colitis. Gut 2006:55:1575-80.

65. Creed TJ, Norman MR, Probert CS, et al. Basilixumab (anti-CD25) in combination with steroids may be an effective new treatment for steroid-resistant ulcerative colitis. Aliment Pharmacol Ther 2003:18:65-75

66. Creed TJ, Probert CS, Norman MN, et al; BASBUC INVESTIGATORS. Basilixumab for the treatment of steroid-resistant ulcerative colitis: further experience in moderate and severe disease. Aliment Pharmacol Ther 2006:23:1435-42.
67. Plevy S, Salzberg B, Van Assche G, et al. A phase I study of visilizumab, a humanized anti-CD3 monoclonal antibody, in severe steroid-refractory ulcerative colitis. Gastroenterology 2007;133:1414-22.

68. Barta Z, Tóth L, Zeher M. Pulse cyclophosphamide therapy for inflammatory bowe disease. World J Gastroenterol 2006;12:1278-80.

69. Lazzerini M, Martelossi S, Marchetti F, et al. Efficacy and safety of thalidomide in children and young adults with intractable inflammatory bowel disease: long-term results. Aliment Pharmacol Ther 2007;25:419-27.

70. Musch E, Andus T, Malek $M$, et al. Successful treatment of steroid refractory active ulcerative colitis with natural interferon-beta-an open long-term trial. Z Gastroenterol 2007:45:1235-40.

71. Stremmel W, Ehehalt R, Autschbach F, et al. Phosphatidylcholine for steroidrefractory chronic ulcerative colitis: a randomized trial. Ann Intern Med 2007:147:603-10.

72. Aoki H, Nakamura K, Yoshimatsu Y, et al. Adacolumn selective leukocyte adsorption apheresis in patients with active ulcerative colitis: clinical efficacy, effects on plasma IL-8, and expression of Toll-like receptor 2 on granulocytes. Dig Dis Sci 2007:52:1427-33.

\section{ANSWERS}

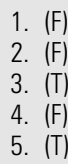

1. $(\mathrm{F}$

5. (T) 